\title{
Drug Transport Mechanisms from Carbopol/Eudragit Verapamil Sustained-Release Tablets
}

e-mail:R.B.Walker@ru.ac.za

\author{
Sandile M. Khamanga and Roderick B. Walker* \\ Faculty of Pharmacy, P.O. Box 94, Rhodes University, Grahamstown, South Africa, 6140
}

\begin{abstract}
The objectives of this study were to compare dissolution profiles of a verapamil (VRP) formulation manufactured inhouse and Isoptin SR using USP Apparatus 2 and 3 and to elucidate drug release kinetics of these dosage forms. Eudragit NE 30D (ethyl acrylate-methyl methacrylate copolymer in a 2:1 ratio) aqueous dispersion was used as a granulating binder for the manufacture of VRP mini-matrix sustained-release tablets. The wet granulation process was performed to prepare free-flowing granules that were blended with Carbopol. The tablets were manufactured using a single-punch press by compression of the granules with magnesium stearate as a lubricant. Drug release was determined in phosphate buffer solution using USP Apparatus 2 and 3. Dissolution data were fitted to zero- and first-order models; in addition, the kinetic data were determined by evaluation of Higuchi release kinetics. The mechanism of drug release was established using the Korsmeyer-Peppas model. In general, all tablets showed high mechanical resistance with less than $1 \%$ friability. There was no significant difference between the dissolution profiles of the formulation manufactured in-house and the commercially available product. The release mechanism of the formulated and marketed products was controlled by anomalous non-Fickian diffusion. VRP release was prolonged for $12 \mathrm{~h}$ indicating the usefulness of the formulation as a twice-daily dosage form. The mechanism of drug release for the dosage forms was unaffected by the choice of apparatus.
\end{abstract}

\section{INTRODUCTION}

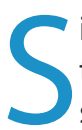
ince the adoption of dissolution testing, pharmaceutical scientists have naturally performed comparisons of dissolution profiles. Many of the methods of comparison have often been qualitative or semiquantitative and have often relied on the technological skill and learned art of the pharmaceutical scientist. While these qualitative assessments of the degree of dissolution profile similarity have been useful, regulatory agencies have required more objective and quantitative methods to compare dissolution profiles for regulatory purposes (1).

In addition, mathematical modeling of drug-release profiles from controlled drug delivery devices has been used to provide knowledge relating to mass-transport mechanisms involved in the control of drug release from dosage forms (2). Of the various mechanisms that control drug release, the most important are diffusion, watertriggered transport (swelling) coupled with chain relaxation, and slow erosion of polymer (2-5). However, the occurrence of multicomponent transport processes, different types of matrices, composition, device geometries, drug loading and saturation solubility in the matrix, diffusion, swelling, polymer dissolution, and erosion may complicate the analysis of drug release from controlledrelease delivery systems (6).

Furthermore, mathematical approaches covering all possible chemical and physical processes are not yet available; hence, to describe drug release, there is a need to identify or develop an adequate mathematical theory

*Corresponding author. for specific types of drug delivery systems (2). It is recognized that alternative approaches have been reported; these should be considered and used in combination with conventional methods of analysis that have already been established (6). These new techniques include the use, for example, of artificial neural network (ANN) methodology to predict drug-release profiles from drug delivery systems $(6,7)$ using popular drug carrier materials.

There is a paucity of information pertaining to the transport mechanism and release kinetics of drugs from dosage forms that contain both Eudragit NE 30D and Carbopol 794P NF. The authors (5) investigated the swelling and erosion behavior of VRP from sustainedrelease tablets based on the theory of macromolecular disentanglement. Their results showed that swelling and erosion behavior dictate the kinetics and mechanism of drug release from sustained-release formulations, but one process may predominate over the other because of different polymer characteristics. VRP, a calcium-channel blocker that limits calcium ion entry into cells, is widely used in the management of angina, supraventricular arrhythmias, and hypertension (8).

The objective of this investigation was to compare the dissolution profiles of a VRP formulation manufactured in our laboratory to those for Isoptin SR to elucidate the release kinetics when using USP Apparatus 2 and 3. In this study Eudragit NE 30D, a neutral milky-white aqueous dispersion of low viscosity and characteristic aromatic odor consisting of polymethacrylic acid esters (9), was used as the granulating agent. Traditionally this material has been used as an aqueous coating agent. 


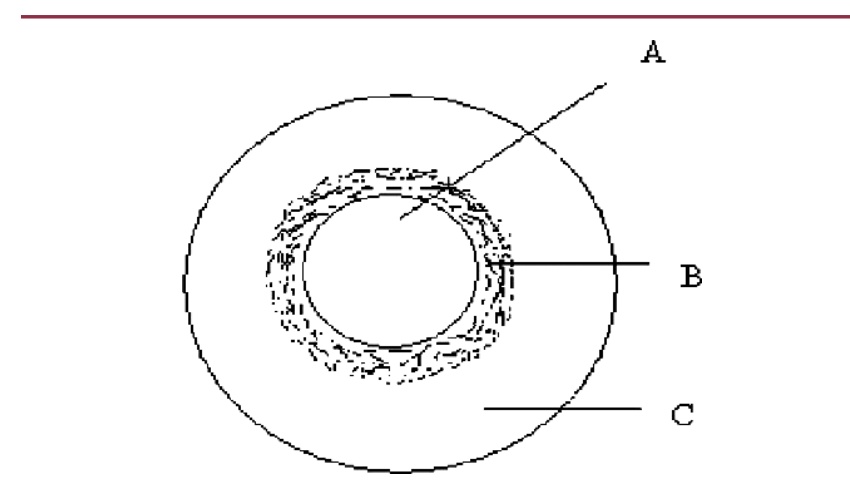

Figure 1. Schematic diagram of a VRP matrix tablet showing $(A)$ inner core of drug-polymer, (B) Eudragit NE 30D, and (C) polymer-filler-lubricant.

\section{MATERIALS AND METHODS \\ Materials}

VRP was used as the model drug in these studies. The drug was supplied by Aspen-Pharmacare (Port Elizabeth, $\mathrm{SA})$. The following materials were used as received: Carbopol 974 PNF (Noveon, Inc., Brecksville, Cleveland, USA); Eudragit NE 30D (Rohm Pharma Polymers, Darmstadt, GmBH); Emcompress (Penwest Pharmaceutical Co., Mendel, UK); Emcocel 90M (Penwest Pharmaceutical Co., Mendel, UK); Ethocel 10 FP (Dow Chemical Co., Michigan, USA); magnesium stearate; and clear size 00 gelatin capsules (Aspen Pharmacare, Port-Elizabeth, SA). All other reagents were at least of analytical grade and were used without further purification.

\section{Methods}

Preparation of the Matrix Tablets

Mini-tablets weighing $240 \mathrm{mg}$, which contained $80 \mathrm{mg}$ VRP, were compressed using a Manesty F3 single-punch tablet press (Manesty Machines Ltd., Liverpool, UK). A set of 7.0-mm, flat-faced edge punches were used to produce the matrix mini-tablets of $4.5 \mathrm{~mm}$ height with a mean crushing strength of about $120.0 \mathrm{~N}$ (Erwerka TBH 28 Tablet Hardness Tester, FRG). Before dissolution testing, three mini-tablets were enclosed in a size 00 hard gelatin capsule to produce a dosage form that contained $240 \mathrm{mg}$ VRP.

Drug-release profiles for test formulation V1 were compared with those of a commercially available product, Isoptin SR, containing $240 \mathrm{mg}$ verapamil as the hydrochloride salt. Batch V1 (5) was prepared by mixing VRP, Carbopol 974P NF, Ethocel 10 FP using a stainless steel Kenwood blender. The powders were weighed separately using a top-loading electronic balance Model PM 4600 (Mettler, Zurich, Switzerland), screened, and granulated with Eudragit NE 30D using a Kenwood planetary mixer (Kenwood, UK). The wet mass was then passed through a mesh screen no. 20 using an oscillating granulator (Erwerka, GmbH, Germany) set at 50 rpm. The granules were dried in a Gallenkamp drying oven maintained at $40^{\circ} \mathrm{C}$ for 12 hours, after which they were rescreened. The granules were then added to Carbopol 974P NF, Ethocel 10 FP, Emcompress, and Emcocel 90M and blended.
Table 1. Wet Granulation Formula of Tablet Batch V1

\begin{tabular}{llc}
\hline & \multicolumn{1}{c}{ Ingredients } & $\mathbf{1} \%(\mathbf{w} / \mathbf{w})$ \\
\hline $\mathbf{1}$ & VRP & 33.0 \\
\cline { 2 - 3 } & Carbopol 974P NF & 5.0 \\
\cline { 2 - 3 } & Ethocel NF & 17.5 \\
\hline $\mathbf{2}$ & Eudragit NE 30D & 3.0 \\
\hline & Carbopol 974P NF & 5.0 \\
\cline { 2 - 3 } & Ethocel NF & 6.0 \\
\cline { 2 - 3 } & Emcocel 90M & 10.0 \\
\cline { 2 - 3 } & Emcompress & 20.0 \\
\hline $\mathbf{4}$ & Magnesium stearate & 0.5 \\
\hline
\end{tabular}

Blending was performed in a 1-kg capacity, cube blender set at a horizontal angle at $100 \mathrm{rpm}$ for $30 \mathrm{~min}$ to ensure adequate mixing. Magnesium stearate was added to the mixture, which was blended for a further $3 \mathrm{~min}$.

Figure 1, a schematic representation of the inner granulate core coated with a polymeric dispersion and the outer core of the matrix tablet, provides a theoretical representation of the behavior of the formulation. The quantitative and qualitative composition of the formulation is shown in Table 1.

\section{In Vitro Dissolution Studies}

In vitro drug-release studies were performed using USP Apparatus 3 (VanKel Industries, New Jersey, USA) as described (10) to determine the effect of continuous $\mathrm{pH}$ change over time on drug-release characteristics. A model VK 750D digitally controlled water circulation/heater (VanKel Industries, New Jersey, USA) was used to maintain the temperature of the dissolution media of different buffers of different $\mathrm{pH}$ values ( $180 \mathrm{~mL}$ per vessel) at $37 \pm 0.5^{\circ} \mathrm{C}$. Drug release for each formulation $(n=6)$ was assessed for $1 \mathrm{~h}$ at $\mathrm{pH} 1.6,1 \mathrm{~h}$ at $\mathrm{pH} 3.4,4 \mathrm{~h}$ at $\mathrm{pH} 4.6,4 \mathrm{~h}$ at $\mathrm{pH} 6.8$, and $8 \mathrm{~h}$ at $\mathrm{pH} 7.4$. A standard oscillation rate of 20 dips per min (dpm) was maintained throughout the studies. Each sample was filtered through a $0.45-\mu \mathrm{m}$ Durapore membrane HVLP filter (Millipore Corporation, Ireland) before analysis. A summary of the dissolution test conditions for these studies is found in Table 2. The samples were analyzed using a high performance liquid chromatography (HPLC) method that had been developed and validated in our laboratory (11). The percent drug release was determined at $278 \mathrm{~nm}$ using a modular HPLC system consisting of a SpectraSERIES P100 pump (ThermoSeparation Products, San Jose, California, USA) and an automated Waters Intelligent Sample Processor Model 710B (WISP, Waters Associates, Milford, MA, USA).

A fully automated Hanson Research SR 8 PLUS (Chartsworth, CA, USA) dissolution apparatus fitted with an Autoplus Multifill and Maximizer Syringe Fraction 
Table 2. Dissolution Test Conditions for USP Apparatus 2 and 3

\begin{tabular}{|c|c|c|}
\hline Parameter & USP Apparatus 2 & USP Apparatus 3 \\
\hline Dissolution medium & $\begin{array}{c}\text { Buffers ( } \mathrm{pH} 1.6, \\
6.8, \text { and } 7.4 \text { ) }\end{array}$ & $\begin{array}{c}\text { Buffers (pH 1.6, 3.4, } \\
4.6,6.8 \text {, and 7.4) }\end{array}$ \\
\hline Temperature & $37.0 \pm 0.5^{\circ} \mathrm{C}$ & $37.0 \pm 0.5^{\circ} \mathrm{C}$ \\
\hline Initial volume & $900 \mathrm{~mL}$ & $180 \mathrm{~mL}$ \\
\hline Paddle / dip speed & $50 / 100$ rpm & $20 \mathrm{dpm}$ \\
\hline Screen size & & $\begin{array}{c}405 \mu \mathrm{m} \text { top } / 177 \mu \mathrm{m} \\
\text { bottom }\end{array}$ \\
\hline Filter size & $0.45 \mu \mathrm{m}$ & $0.45 \mu \mathrm{m}$ \\
\hline Volume drawn & $2 \mathrm{~mL}$ & $2 \mathrm{~mL}$ \\
\hline \multirow[t]{3}{*}{ Dissolution time } & \multirow{3}{*}{$\begin{array}{c}1 \mathrm{~h} \text { in } \mathrm{pH} 1.6 \\
21 \mathrm{~h} \text { each in } \mathrm{pH} 6.8 \\
\text { and } 7.4\end{array}$} & $\begin{array}{l}1 \mathrm{~h} \text { each in } \mathrm{pH} 1.6 \\
\text { and } 3.4\end{array}$ \\
\hline & & $4 \mathrm{~h}$ each in $\mathrm{pH} 4.6,6.8$ \\
\hline & & $8 \mathrm{~h}$ in $\mathrm{pH} 7.4$ \\
\hline
\end{tabular}

Collector was used as USP Apparatus 2. The dissolution medium was $900 \mathrm{~mL}$ of $0.1 \mathrm{M}$ phosphate buffer ( $\mathrm{pH} 7.4$ ) maintained at $37 \pm 0.5^{\circ} \mathrm{C}$, and the paddles stirred at 100 rpm. Sink conditions were used in the experiment, and aliquots were withdrawn at $0,1,2,6,10,14$, and $22 \mathrm{~h}$ and analyzed using a validated isocratic HPLC method (11). A summary of the dissolution test conditions for these studies is depicted in Table 2.

Drug-release data were fitted to different release models. Experimental results were expressed as a mean \pm standard deviation (SD). In this study, two fit factors that compare the dissolution profiles of a pair of drug products were applied to the dissolution data. These fit factors directly compare the difference between percent drug dissolved per unit time for a test and a reference product. The fit factors are denoted $f_{1}$ (difference factor), and $f_{2}$ (similarity factor) and are defined by Moore and Flanner (12). Because $f_{2}$ values are sensitive to the number of dissolution time points, only one measurement should be considered after $85 \%$ dissolution of the product. One-way analysis of variance (ANOVA) followed by Bonferroni's post hoc test was applied to check for significant difference in drug release from the dosage forms at different time points. Differences were considered statistically significant at $p<0.05$. The statistical software used for these analyses was GraphPad Prism Software Version 5.02 (GraphPad Prism Software, San Diego, CA, USA). The advantage of using ANOVA is that there is no restriction to any of the requirements as suggested by Moore and Flanner with the description of the fit factor, $f_{2}$, and it does not depend on fitting data to a specific kinetic model (12).

\section{Assay}

Twenty randomly selected VRP tablets were weighed and powdered using a mortar and pestle. An aliquot of powder equivalent to the weight of three tablets or $240 \mathrm{mg}$ of drug was accurately weighed and transferred into a $100-\mathrm{mL}$ volumetric flask containing $70 \mathrm{~mL}$ mobile phase (11). The solution was stirred continuously for $1 \mathrm{~h}$ using a magnetic stirrer (Gallenkamp ${ }^{\mathrm{TM}}$, UK) and then made up to volume with mobile phase. The solution was filtered through a 0.45- $\mu$ m hydrophilic PVDF (Millipore Millex-HV, Millipore Corporation, Bedford, MA, USA) membrane before analysis by HPLC. This procedure was repeated in triplicate and the average value calculated.

\section{Uniformity of Weight and Thickness}

Twenty tablets were randomly selected from each batch and weighed using a top-loading electronic balance Model AG 135 (Mettler Toledo, Switzerland), and the average weight of each manufactured batch established. The average weight and the standard deviation of each were then calculated.

\section{Crushing Strength}

The crushing strength of twenty randomly selected tablets was measured using an Erweka TBH 28 hardness tester (Erweka, GmbH, Heusenstamm, Germany) $24 \mathrm{~h}$ after compaction.

\section{Friability}

The friability of all batches of tablets was determined using an Erweka TA3R friabilator (Erwerka, GmbH, Heusenstamm, Germany). Twenty tablets were dedusted and weighed on an electronic top-loading balance Model AE 163 (Mettler, Zurich, Switzerland). The tablets were allowed to tumble for $4 \mathrm{~min}$ at $25 \mathrm{rpm}$ or for 100 drop cycles. Tablets were dedusted and reweighed, and the percent friability of the tablets was calculated.

\section{Release Models}

The drug-release data were fitted to different models in an attempt to establish the kinetics and release mechanisms of VRP from these dosage forms. Various methods of analyses that have been described are separated into model-dependent and independent methods (13). The mathematical models that were used to describe the dissolution curves are shown in Table 3.

The release data were analyzed using zero-order (14), first-order (15), and Higuchi $(16,17)$ models. The dissolution profiles obtained from batch V1 and Isoptin SR products were compared by the determination of the $f_{1}$ and $f_{2}$ factors. The equivalence of two profiles is observed when $f_{1}$ values are between 0 and 15 and $f_{2}$ values are between 50 and 100 (12). Modeling was limited to three models that are commonly applied to drug-release data from solid dosage forms. In each case, model fitting was accomplished using GraphPad Prism Software Version 5.02 (GraphPad Prism Software, San Diego, CA, USA). Goodness of fit was the primary criterion for selecting the most appropriate model that described the data generated in these studies $(18,19)$. 
Table 3. Mathematical Representation of Models used to Describe the Release Profiles of Batch V1 and Isoptin SR

\begin{tabular}{lc}
\hline Model & Equation \\
\hline Zero-order & $Q_{t}=Q_{o}+K_{o} t$ \\
\hline First-order & $\operatorname{Ln} Q_{t}=\operatorname{Ln} Q_{o}+K_{l} t$ \\
\hline Higuchi & $Q_{t}=K_{H} t^{1 / 2}$ \\
\hline
\end{tabular}

To assess the impact of a constant-pH dissolution medium on the kinetic rate constant, dissolution testing was also performed in media of $\mathrm{pH} 1.6,4.6,6.8$, and 7.4 individually using USP Apparatus 2. To analyze the mechanism of drug release from these dosage forms, the dissolution data were fitted to Korsmeyer-Peppas equation (20) shown in eq 1 and other models as shown in Table 3.

$$
\frac{M_{t}}{M_{\infty}}=K t^{n}
$$

where $M_{t}$ represents the fraction of drug released in time $t$, and $M_{\infty}$ is the amount of drug released after an infinite time. $K$ represents a constant incorporating structural and geometrical characteristic of the device, and $n$ is the diffusional exponent of drug release that characterizes the type of release mechanism during the dissolution process. For non-Fickian release, the $n$ value falls between 0.5 and 1.0, while for Fickian diffusion, $n=0.5$; for zero-order release (case II transport), $n=1$;

and for super-case II transport, $n>1$.

\section{RESULTS AND DISCUSSION}

The manufacture of the granules posed several difficulties when processing with these polymers. Initially, drying was conducted at $60^{\circ} \mathrm{C}$, but the granules were tacky. The

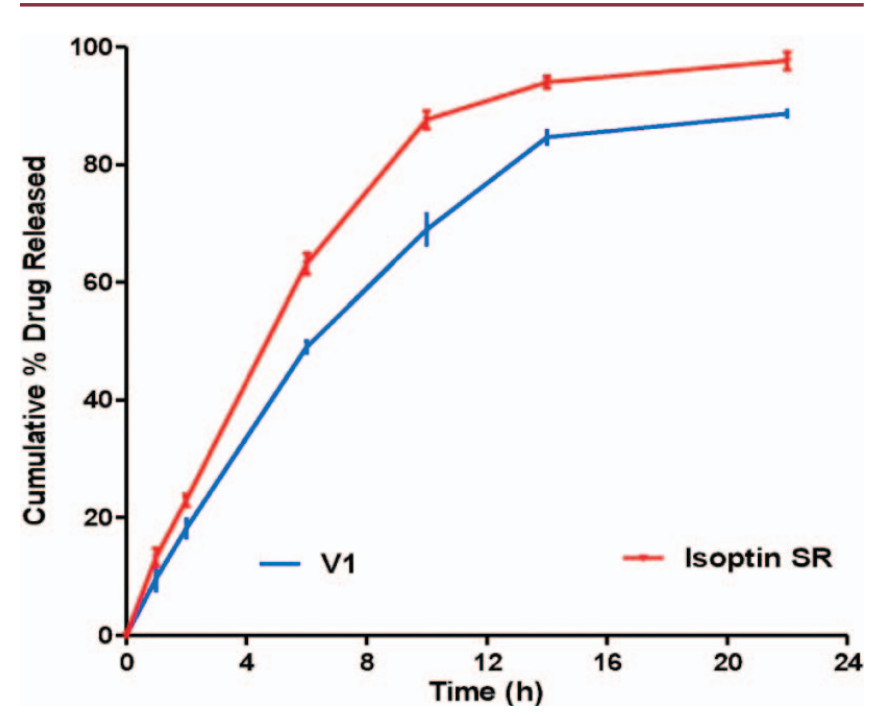

Figure 2. Dissolution profiles of verapamil hydrochloride from tablets of batch V1 and Isoptin SR (Mean $\pm S D, n=6$ ) using USP Apparatus 2.

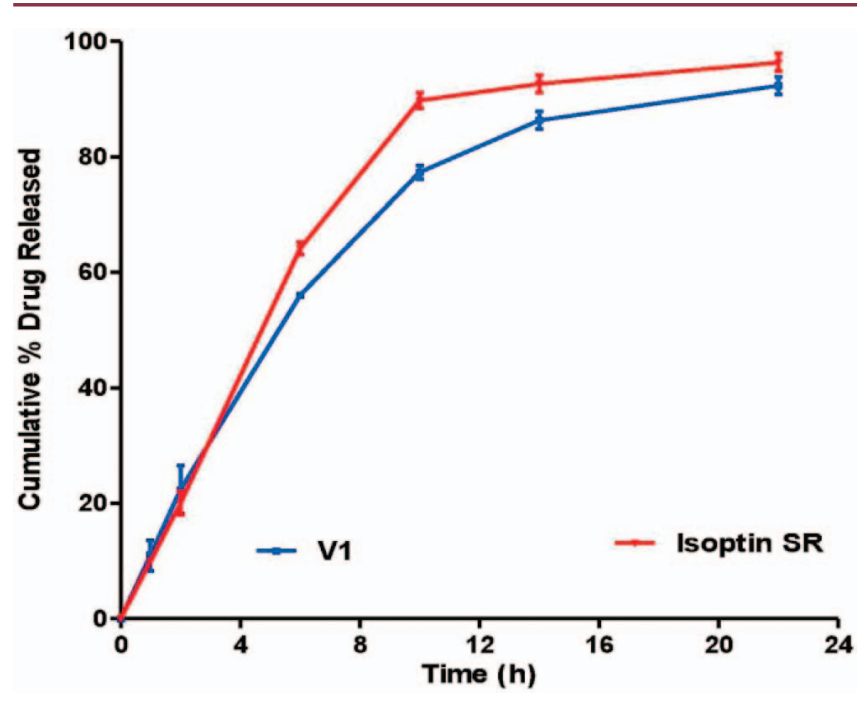

Figure 3. Dissolution profiles of verapamil hydrochloride from tablets of batch V1 and Isoptin SR (Mean $\pm S D, n=6$ ) using USP Apparatus 3.

thermal stability of Eudragit and Carbopol has been investigated by microscopic reflectance Fourier transform infrared spectroscopy. Results indicate the formation of a 6-membered ring cyclic anhydride product in the molecular structures of Eudragit and Carbopol polymers via inter- or intrapolymer condensation when the temperature is increased (21). Therefore mechanical changes possibly occurred when the temperature was raised, thus damaging or deforming the polymers. These may ultimately have resulted in changes in dissolution properties of the drug after storage. As a result, the drying process was maintained at $40^{\circ} \mathrm{C}$, and a glue-like, hard homogeneous mass formed and passed through the wire mesh \#20 screen with difficulty. Consequently, this caused abrasion of the mesh wires of the screen with associated low efficiency and productivity. On compression, the tablets that were formed had smooth surfaces and did not stick to the punches. The formulation using the granulating agent resulted in a very wet mass that required drying for about $12 \mathrm{~h}$.

\section{Tablet Characterization}

All mini-tablets met the pharmacopeial specifications for weight variation, content uniformity, and friability. In general, all tablets showed high mechanical resistance with less than $1 \%$ friability, and the average assay result was between $98.21 \%$ and $99.62 \%$ for VRP.The tablets
A

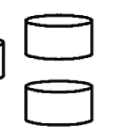

Single units

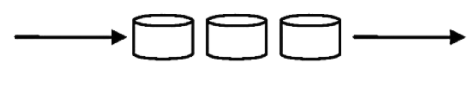

Association

\section{C}

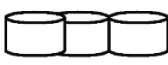

Single rod
Figure 4. Mini-matrix tablets adhering together to form rod-like cylinders during dissolution testing. 
Table 4. Summary of Korsmeyer-Peppas Best-Fit Parameters for Batch V1 and Isoptin SR in Dissolution Media of Different pH Using USP Apparatus 2

\begin{tabular}{lcccc}
\hline Formulation & $\mathbf{p H}$ & $\begin{array}{c}\text { Kinetic } \\
\text { constant }(\boldsymbol{K})\end{array}$ & $\begin{array}{c}\text { Release } \\
\text { exponent }(\boldsymbol{n})\end{array}$ & $\begin{array}{c}\text { Coefficient of } \\
\text { determination }\left(\boldsymbol{R}^{2}\right)\end{array}$ \\
\hline $\mathrm{V} 1$ & 1.6 & 14.67 & 0.7989 & 0.9982 \\
\cline { 2 - 5 } & 4.6 & 2.155 & 0.9614 & 0.9859 \\
\cline { 2 - 5 } & 6.8 & 1.263 & 1.1711 & 0.9698 \\
\cline { 2 - 5 } & 7.4 & 8.233 & 0.8969 & 0.9689 \\
\hline Isoptin SR & 1.6 & 14.96 & 0.7054 & 0.9955 \\
\cline { 2 - 5 } & 4.6 & 7.861 & 0.9047 & 0.9817 \\
\cline { 2 - 5 } & 6.8 & 9.083 & 0.8944 & 0.9997 \\
\cline { 2 - 5 } & 7.4 & 7.726 & 1.0054 & 0.9982 \\
\hline
\end{tabular}

showed some variation in weight, but none of the tablets varied by more than $5 \%$ of the mean weight. Similarly, the thickness of the tablets was uniform. Although the tests for these tablet parameters are not official, they suggest that the flow properties of the granulation before tableting were adequate (22) because changes in the thickness will eventually affect other tablet parameters such as drug content and weight.

\section{In Vitro Dissolution Studies}

The plots of percent drug released versus time for batch V1and Isoptin SR are depicted in Figures 2 and 3. When placed in the dissolution medium, the gelatin capsules quickly dissolved in minutes. The remaining tablets, which were now fully exposed to the dissolution medium, swelled. The matrix tablets did not maintain their original shape throughout dissolution testing, indicating that swelling was also likely contributing to the process of drug release (5).

Drug release from these matrix tablets exhibited an almost linear release profile in the initial stages of dissolution. A progressively slower release was observed with time because the polymers used had started to swell. As seen in Figures 2 and 3, at the end of the dissolution test, drug release was less than $100 \%$, which was not a function of a low assay value. The dissolution profiles observed from USP Apparatus 3 (Figure 3) exhibited a faster dissolution rate than the dissolution profiles from USP Apparatus 2 (Figure 2). This is most likely caused by the different hydrodynamic conditions that exist in the cylinders used in this apparatus. There is variability in the fluid velocity with position in the different vessels that are used during dissolution testing. Fluid shear rates also vary because of the hemispherical vessel (inverted cupola as used in architectural geometry) used in USP Apparatus 2 and the flat-based cylinder that is used in USP Apparatus 3.

At a reciprocation rate of $20 \mathrm{dpm}$, turbulent flow may be generated in USP Apparatus 3. This may cause chaotic flow and complex fluid dynamics within the dissolution vessel and in turn weaken the gel structure that immediately forms around a tablet at the commencement of hydration (10) resulting in an increase in VRP release.

The difference in release rates between the two formulations could be due in part to the possible

Table 5. Summary of Korsmeyer-Peppas Best-Fit Parameters for Batch V1 and Isoptin SR in Dissolution Media of Different pH Using USP Apparatus 3

\begin{tabular}{|c|c|c|c|c|c|c|}
\hline Formulation & $\frac{M_{t}}{M_{\infty}}$ & Time (h) & pH & Kinetic constant $(K)$ & Release exponent $(n)$ & $\begin{array}{c}\text { Coefficient of } \\
\text { Determination }\left(R^{2}\right)\end{array}$ \\
\hline \multirow[t]{6}{*}{ V1 } & & 1 & 1.6 & & & \\
\hline & 0.19 & 2 & 1.6 & & & \\
\hline & 0.57 & 6 & 4.6 & 12.06 & 0.8409 & 0.9794 \\
\hline & 0.80 & 10 & 6.8 & 12.11 & 0.8265 & 0.9890 \\
\hline & 0.84 & 14 & 7.4 & 12.56 & 0.7693 & 0.9809 \\
\hline & 0.84 & 22 & 7.4 & & & \\
\hline \multirow[t]{6}{*}{ Isoptin SR } & & 1 & 1.6 & & & \\
\hline & 0.18 & 2 & 1.6 & & & \\
\hline & 0.64 & 6 & 4.6 & 9.490 & 1.0468 & 0.9938 \\
\hline & 0.92 & 10 & 6.8 & 9.700 & 0.9998 & 0.9949 \\
\hline & 0.94 & 14 & 7.4 & 10.04 & 0.9138 & 0.9787 \\
\hline & 0.94 & 22 & & & & \\
\hline
\end{tabular}




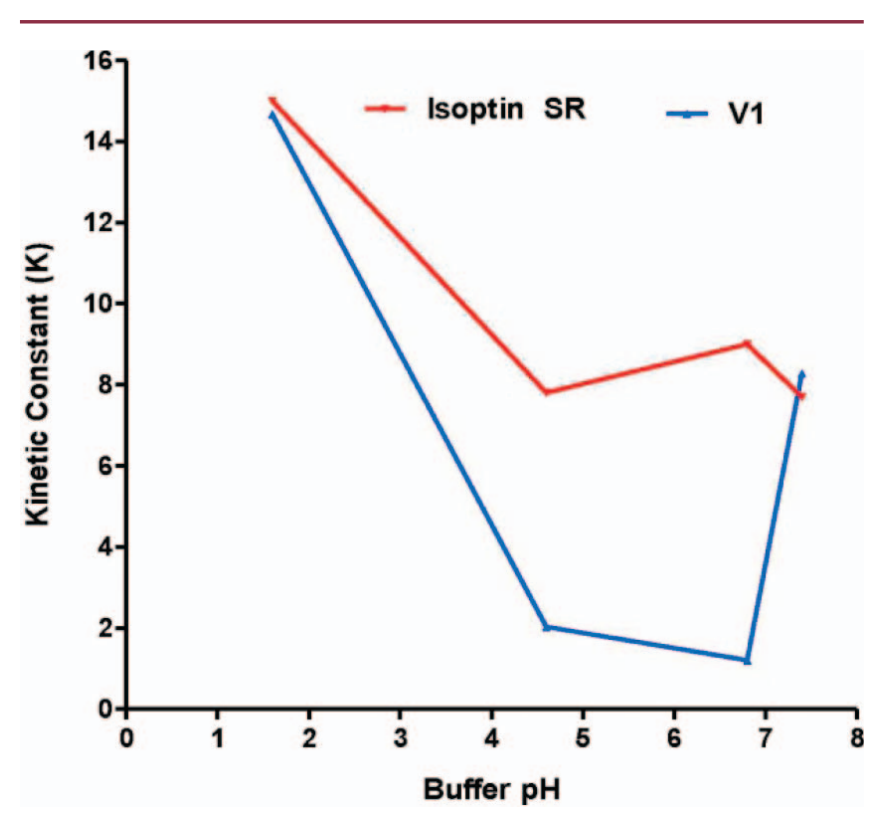

Figure 5. pH Effect on the kinetic constant of batch V1 and Isoptin SR.

interaction between the cationic VRP and the anionic polymer, Carbopol. Interaction may not be evident in the solid state, but cannot be avoided when the dosage form is exposed to the dissolution medium (23). Such observations have also been noted when a cationic drug was incorporated with an anionic surfactant (24) and a polymer (25).

The degree of swelling increased when the dissolution medium was $\mathrm{pH} 7.4$ as opposed to $\mathrm{pH}$ 1.6. As the hydration and swelling progressed, the mini-tablets rapidly formed a single rod-like cylinder, thus adhering to one another as shown in Figure 4. Thereafter, a lower surface area was exposed to the dissolution medium than when the mini-tablets were separate entities.

\section{Effect of pH}

The values of $n$ as estimated by non-linear regression are shown in Tables 4 and 5. The experimental values of $n$ lie between 0.7 and 1.0 for all formulations tested, indicating that non-Fickian release kinetics predominate and drug-release mechanisms involve a combination of diffusion and polymer chain-relaxation mechanisms. The contribution of these mechanisms to the overall release is considered additive.

A plot of kinetic constant versus pH is shown in Figure 5, and the best-fit model parameters are listed in Table 6. At a $\mathrm{pH}$ of 1.6, the average values of $K$ were high, and when the $\mathrm{pH}$ was increased to 4.6 , the rate constants decreased to a minimum value after which the release rate constants increased. Conversely, the $K$ values for Isoptin SR tablets remained relatively unchanged as the $\mathrm{pH}$ was changed from 4.6 to 7.4 .

The lowest average $K$ occurred at $\mathrm{pH} 6.8$ for batch $\mathrm{V} 1$. There is a direct relationship between total percent drug released and $K$. The decrease in kinetic rate constants observed for batch $\mathrm{V} 1$ at higher $\mathrm{pH}$ values may be explained in part by the increase in the diffusional path length within the dosage form, through which the drug must pass before release.

It was observed (5) that swelling was dominant in this formulation and that the polymer developed a highly viscous gel when exposed to the dissolution media. On the contrary, the effect was not observed for Isoptin SR tablets. This finding confirms that when the $\mathrm{pH}$ is increased from 1.6 to 6.8 , there is an increased dissociation of polymer carboxylic groups, which facilitates interaction of the anionic carboxyl group with the basic amine nitrogen of VRP.The effect of the decreased drug solubility with increasing $\mathrm{pH}$ is not very significant, as seen for Isoptin SR tablets, which were not affected. Many authors $(23,26)$ observed an increase in the interaction of anionic polymers and cationic drugs with increasing $\mathrm{pH}$.

Furthermore, VRP is a salt of a weak base and a strong acid and shows $\mathrm{pH}$-dependent solubility characteristics (27). Based on its $\mathrm{p} K_{\mathrm{a}}(\approx 8.8)(28)$, VRP deprotonates in intestinal fluid ( $\mathrm{pH} 7.4$ ), resulting in the formation of a nonionized form, verapamil base (V). According to the Henderson-Haselbach equation, the [VRP]/[V] ratio is relatively high $(\approx 1: 0.04)$ at these conditions. Verapamil base has significantly lower solubility, and thus drug dissolution would be expected to be lower with a corresponding decrease in $K$ values.

Table 6. Resultant Model Parameters from Fitting Dissolution Data Obtained Using USP Apparatus 2 and 3 for Batches V1 and Isoptin SR

\begin{tabular}{|c|c|c|c|c|c|c|c|c|}
\hline \multirow[t]{3}{*}{ Formulation } & \multirow{3}{*}{$\begin{array}{c}\text { USP } \\
\text { Apparatus }\end{array}$} & \multirow[b]{3}{*}{ pH } & \multicolumn{6}{|c|}{ Model Parameters } \\
\hline & & & \multicolumn{2}{|c|}{ Zero-order } & \multicolumn{2}{|c|}{ First-order } & \multicolumn{2}{|c|}{ Higuchi } \\
\hline & & & $R^{2}$ & $k_{\text {o }}$ & $R^{2}$ & $k_{1}$ & $R^{2}$ & $\boldsymbol{k}_{\mathrm{H}}$ \\
\hline \multirow[t]{2}{*}{ V1 } & 3 & 7.4 & 0.9357 & 6.2862 & 0.6380 & 0.2415 & 0.9638 & 25.2580 \\
\hline & 2 & 7.4 & 0.8758 & 0.3684 & 0.7858 & 0.1122 & 0.9619 & 21.8300 \\
\hline \multirow[t]{2}{*}{ Isoptin SR } & 3 & 7.4 & 0.9300 & 7.3119 & 0.6828 & 0.2609 & 0.9459 & 29.1960 \\
\hline & 2 & 7.4 & 0.8147 & 0.5074 & 0.8146 & 0.2020 & 0.9416 & 24.3401 \\
\hline
\end{tabular}


Table 7. Comparison of Percent Dissolved Drug Using Moore's Difference and Similarity Factors

\begin{tabular}{lcc}
\hline Comparison & $\boldsymbol{f}_{\mathbf{1}}$ (Difference) & $\boldsymbol{f}_{\mathbf{2}}$ (Similarity) \\
\hline USP 2 vs USP 3 (V1) & 5.3 & 68.3 \\
\hline USP 2 (V1 vs Isoptin SR) & 14.3 & 52 \\
\hline USP 3 (V1 vs Isoptin SR) & 9.4 & 59.4 \\
\hline
\end{tabular}

Carbopol 974P NF has a $\mathrm{p} K_{\mathrm{a}}$ of approximately 6.0. At pH values above the $p K_{a}$, the polymer chains and the carboxylate moiety on the polymer start to ionize (29), resulting in repulsion between the negative charges, which favors the swelling of the polymer. At this stage, interaction of the polymer and VRP occurs, and an insoluble complex is likely formed, which further retards release.

In addition, the VRP-Carbopol complex, with a lower solubility than VRP, results in a decrease in the dissolution of VRP from batch V1. This complex formed by a charge interaction that could have decreased the tortuosity and reduced the porosity of the matrix system, which affect drug release from these systems. To study the possible interaction between VRP and the anionic polymer, precipitation titration tests were performed using the Wells and Parrot method (30). No change occurred in a VRP solution when mixed with dissolution medium. On addition of the polymer, the solution became cloudy or milky (due to complex formation) and turned clear when a high concentration of the polymer was added.

In addition, at $\mathrm{pH}$ values above the $\mathrm{p} K_{\mathrm{a}}$, the carboxylic acid groups of Carbopol are more likely to be dissociated, and repulsion of the chains due to these charges may cause expansion of the molecule, which can form a gel that slows drug-release rates. The sudden dip in the curves depicted in Figure 5 at $\mathrm{pH} 6.8$ may be explained in part by this phenomenon. It has been reported (31-33) that there is a linear relationship between the release rate of a drug and the degree of interaction between the polymers.

Commercial Isoptin SR tablets contain hypromellose, macrogols, sodium alginate, and povidone (34) as retarding polymers, while V1 contains Carbopol, Ethocel, and a granulating agent. Therefore, these differences in $K$ values are likely caused largely by the interaction between the carboxylic group of the polymer and the tertiary amine of the drug. In addition, the thick coat of polymer-granulating agent around the drug (Figure 1) produces strong binding among granules.

The results of the analysis of dissolution data are shown in Table 6. Higuchi and zero-order models seemed to fit the dissolution data of V1 and Isoptin SR adequately. Fitting the drug-release data to a zero-order model revealed $K_{\circ}$ (rate constant) values of $0.37-6.3$ for batch $V 1$ and $0.50-7.31$ for Isoptin SR. A two-sided $t$-test conducted at the $95 \%$ level of significance $(\alpha=0.05)$ revealed that there was no statistically significant difference $(p>0.05)$ between the $K_{\mathrm{o}}$ values obtained for the different formulations in all cases. A similar trend was observed when the data were fitted to the Higuchi model. There were no statistically significant differences $(p>0.05)$ between the $K_{\mathrm{H}}$ values obtained for the different formulations.

The difference $\left(f_{1}\right)$ and similarity $\left(f_{2}\right)$ factors (12) were also calculated. No differences between the dissolution profiles of the formulated and reference products were observed when using $f_{2}$ to evaluate the dissolution data generated using both test systems (Table 7). A comparison of USP Apparatus 2 and 3 resulted in an $f_{2}$ value of 68.3 for the test product. All other results when comparing the test to the reference product showed similarities. The similarity factor $f_{2}$ has been endorsed by the FDA as an acceptable method for dissolution profile comparison (35). The results show that USP Apparatus 2 and 3 lack the sensitivity to discriminate between dissolution profiles, as can be seen from these results.

Table 8. Comparison (Bonferroni post-test) of Percent Dissolved Drug at Each Time Point from Formulated Product and Isoptin SR in USP Apparatus 2 and 3

\begin{tabular}{|c|c|c|c|c|c|c|}
\hline & $\begin{array}{c}\text { USP Apparatus } 2 \text { vs } 3 \\
\text { (V1 vs V1) }\end{array}$ & & $\begin{array}{l}\text { USP Apparatus } 2 \\
\text { (V1 vs ISOPTIN) }\end{array}$ & & $\begin{array}{l}\text { USP Apparatus } 3 \\
\text { (V1 vs ISOPTIN) }\end{array}$ & \\
\hline Time (h) & $P$ value & Summary & $P$ value & Summary & $P$ value & Summary \\
\hline 0 & $>0.05$ & $\mathrm{~ns}^{a}$ & $>0.05$ & ns & $>0.05$ & ns \\
\hline 1 & $>0.05$ & ns & $<0.001$ & ns & $<0.001$ & ns \\
\hline 2 & $>0.05$ & ns & $<0.001$ & ns & $<0.001$ & ns \\
\hline 6 & $>0.05$ & ns & $<0.001$ & $* * *$ & $<0.001$ & $* * *$ \\
\hline 10 & $>0.05$ & ns & $<0.001$ & $* * *$ & $<0.001$ & $* * *$ \\
\hline 14 & $<0.001$ & $* * *$ & $<0.001$ & $* * *$ & $<0.001$ & $* * *$ \\
\hline 22 & $<0.001$ & $* * *$ & $<0.001$ & $* * *$ & $>0.05$ & ns \\
\hline
\end{tabular}

${ }^{a}$ not signi ficant. 
No differences in drug release were observed at any time points except 14 and $22 \mathrm{~h}$ (Table 8) when the data were analyzed using the Bonferroni post-hoc test. The results did not emphasize the sensitivity and discriminative capacity of dissolution performance of the formulated product to Isoptin SR. In the present study, the apparatus were not discriminative when using the statistical tests to evaluate the dissolution profiles.

\section{CONCLUSION}

The results show that with the appropriate paddle speed, USP Apparatus 2 can produce dissolution profiles similar to those obtained with USP Apparatus 3 or distinguish dissolution characteristics to serve the purpose of product control. Our studies also indicate that the release mechanisms of VRP were similar regardless of which apparatus was used for dissolution testing. There was a good fit of drug-release pattern to the Higuchi and zeroorder models, and these have been used to compare the performance of test product with that of an innovator product. The complexity of this formulation and the components used in sustained-release products indicate that drug release is controlled by more than one process, and the effects of formulation composition and test methodology on drug release must be thoroughly investigated in formation development studies. The similarity factor alone was not sufficient to describe the entire dissolution profiles or to discriminate between V1 and Isoptin SR at all time points. Statistical analysis using the Bonferroni post-hoc test was applied to locate the differences in percent released at specific time points between the formulations. These helped in better understanding the dissolution profiles and enabled comparison of the formulations from $t=1 \mathrm{~h}$ to $t=22 \mathrm{~h}$.

\section{ACKNOWLEDGMENTS}

The authors would like to thank the NRF (RBW), Rhodes University (SMMK, RBW), and the Andrew W. Mellon Foundation (SMMK). Rohm Pharma and Aspen Pharmacare are gratefully acknowledged for their donation of excipients.

\section{REFERENCES}

1. Polli, J.E.; Rekhi, G. S.; Shah, V.P. Methods to compare dissolution profiles. Drug Inf. J. 1996, 30, 1113-1120.

2. Siepmann, J.; Peppas, N. A. Mathematical modeling of controlled drug delivery. Adv. Drug Del. Rev. 2001, 48 (2-3), 137-138.

3. Huang, L. L.; Schwartz, J. B. Studies on drug release from a carbomer tablet matrix. Drug Dev. Ind. Pharm. 1995, 21 (13), 1487-1501.

4. Agarwal, V.; Mishra, B. Design, development, and biopharmaceutical properties of buccoadhesive compacts of pentazocine. Drug Dev. Ind. Pharm.1999, 25 (6), 701-709.

5. Khamanga, S. M.;Walker, R. B. Evaluation of rate of swelling and erosion of verapamil (VRP) sustained-release matrix tablets. Drug Dev. Ind. Pharm. 2006,

32 (10), 1139-1148.

6. Reis, M. A. A.; Sinisterra, R. D.; Belchior, J. C. An alternative approach based on artificial neural networks to study controlled drug release. J. Pharm. Sci. 2004, 93 (2), 418-430.

7. Peng, Y.; Geraldrajan, M.; Chen, Q.; Sun, Y.; Johnson, J. R.; Shukla, A. J. Prediction of dissolution profiles of acetaminophen beads using artificial neural networks. Pharm. Dev.Tech. 2006, 11 (3), 337-349.

8. Eisenberg, M. J.; Brox, A.; Bestawros, A. N. Calcium channel blockers: an update. Am. J. Med. 2004, 116 (1), 35-43.

9. Eudragit NE 30D data sheets. Evonik Industries, Evonik Röhm GmbH, Kirschenallee, Darmstadt, Germany.

10. Khamanga, S.M.M.; Walker, R.B. The Effects of Buffer Molarity, Agitation Rate, and Mesh Size on Verapamil Release from Modified-Release Mini-Tablets Using USP Apparatus 3. Dissolution Technol. 2007, 14 (2), 19-23.

11. Khamanga, S.M. M. Formulation and Assessment of Verapamil Sustained Release Tablets. M.Sc. Thesis, Rhodes University, Grahamstown, South Africa, 2005.

12. Moore, J.W.; Flanner, H. H. Mathematical comparison of curves with an emphasis on in vitro dissolution profiles. Pharm. Tech. 1996, 20 (6), 64-75.

13. Sathe, P. M.; Tsong, Y.; Shah, V. P. In-vitro dissolution profile comparison: Statistics and analysis, model dependent approach. Pharm. Res. 1996, 13 (12), 1799-1803.

14. Donbrow, M.; Samuelov, Y. Zero order drug delivery from double-layered porous films: release rate profiles from ethyl cellulose, hydroxypropyl cellulose and polyethylene glycol mixtures. J. Pharm. Pharmacol. 1980, 32 (7), 463-470.

15. Lapidus, H.; Lordi, N. G. Some factors affecting the release of a water-soluble drug from a compressed hydrophilic matrix. J. Pharm. Sci. 1966, 55 (8), 840-843.

16. Higuchi, T. Rate of release of medicaments from ointment bases containing drugs in suspension. J. Pharm. Sci. 1961, 50 (10), 874-875.

17. Higuchi, T.Mechanism of sustained-action medication. Theoretical analysis of rate of release of solid drugs dispersed in solid matrices. J. Pharm. Sci. 1963, 52 (12), 1145-1149.

18. Costa, P.; Lobo, J. M. S. Modeling and comparison of dissolution profiles. Eur. J. Pharm. Sci. 2001, 13 (2), 123-133.

19. Sood, A.; Panchagnula, R. Drug release evaluation of diltiazem CR preparations. Int. J. Pharm. 1998, 175 (1), 95-107.

20. Korsmeyer, R.W.; Gurny, R.; Doelker, E.; Buri, P.; Peppas, N.A. Mechanisms of solute release from porous hydrophilic polymers. Int. J. Pharm. 1983, 15 (1), 25-35.

21. Shan-Yang, L.;Hui-Ling, Y.Thermal stability of methacrylic acid copolymers of Eudragits L, S, and L30D and the acrylic acid polymer of carbopol.J. Polym.Sci., Part A: Polym. Chem. 1999, 37 (13), 2061-2067.

Dissolution Technologies | AUGUST 2011 
22. Carstensen, J. J.; Ping C. C. J. Flow rates and repose angles of wet-processed granulations.J.Pharm.Sci. 1977, 66 (9), 1235-1239.

23. Elkheshem, S.A. Interaction of verapamil hydrochloride with carbopol 934P and its effect on the release rate of the drug and the water uptake of the polymer matrix.Drug Dev. Ind. Pharm. 2001, 27 (9), 925-934.

24. Daly, P. B.; Davis, S. S.; Kennerly, J.W.The effect of anionic surfactants on the release of chlorpheniramine from a polymer matrix tablet. Int. J. Pharm. 1984, $18(1-2), 201-205$.

25. Sadeghi, F.; Ford, J. L.; Rajabi-Siahboomi, A. The influence of drug type on the release profiles from Sureleasecoated pellets. Int. J. Pharm. 2003, 254 (2), 123-135.

26. Neau, S.H.; Chow, M.Y.; Durrani, M. J. Fabrication and characterization of extruded and spheronized beads containing carbopol 974P, NF resin. Int. J. Pharm. 1996, $131(1), 47-55$.

27. Streubel, A.; Siepmann, J.; Dashevsky, A.; Bodmeier, R. $\mathrm{pH}$-independent release of a weakly basic drug from water-insoluble and -soluble matrix tablets. J. Controlled Release 2000, 67 (1), 101-110.

28. Hasegawa, J.; Fujita, T.; Hayashi, Y.; Iwamoto, K.; Watanabe, J. pKa Determination of verapamil by liquid-liquid partition.J. Pharm. Sci. 2006, 73 (4), 442-445.

29. Jian-Hwa, G. Carbopol Polymers for Pharmaceutical Drug Delivery Applications (Excipient Update). Drug Dev. Deliv. [Online] 2003, 3 (6). http://www. drugdeliverytech.com/ME2/dirmod.asp?sid= 4306B1E9C3CC4E07A4D64E23FBDB232C\&nm= Back+lssues\&type $=$ Publishing\&mod $=$ Publications\%3A\%3AArticle\&mid= 8F3A7027421841978F18BE895F87F791\&tier=4\&id=
C691909D749749438ABEF792F1A24A69 (accessed July 19, 2011).

30. Wells, M. L.; Parrott, E. L. Effect of surfactants on release of a highly water-soluble medicinal compound from an inert, heterogeneous matrix.J. Pharm. Sci. 1992, 81 (5), 453-457.

31. Yuasa, H.;Takahashi, H.;Ozeki, T.; Ueno, M. Application of the solid dispersion method to the controlled release of medicine. III: Control of the release of slightly water soluble medicine from solid dispersion granules. Chem. Pharm. Bull. 1993, 41 (2), 397-399.

32. Yuasa, H.; Takahashi, H.; Ozeki, T.; Kanaya, Y.; Ueno, M. Application of the solid dispersion method to the controlled release of medicine. Vl: Release mechanism of a slightly water soluble medicine and interaction between flurbiprofen and hydroxypropyl cellulose in solid dispersion. Chem. Pharm. Bull. 1994, 42, 354-358.

33. Ozeki, H.; Yuasa, Kanaya, Y. Application of the solid dispersion method to the controlled release of medicine. Difference in the release of flurbiprofen from solid dispersions with poly(ethylene oxide) and hydroxypropylcellulose and the interaction between medicine and polymers. Int. J. Pharm. 1997, 155 (2), 209-217.

34. Isoptin: verapamil hydrochloride. Royal Australian College of General Practitioners. Consumer Medicine Information. http://www.racgp.org.au/cmi/abcisopt. pdf (accessed July 23, 2011).

35. Dissolution Testing of Immediate Release Solid Oral Dosage Forms; Guidance for Industry; U.S. Department of Health and Human Services, Food and Drug Administration, Center for Drug Evaluation and Research (CDER), U.S. Government Printing Office: Washington, DC, 1997. 